\title{
LA RESPONSABILIDAD DE LAS PLATAFORMAS DE ECONO- MÍA COLABORATIVA A LA LUZ DE LA LEY 34/2002 DE SERVICIOS DE LA SOCIEDAD DE LA INFORMACIÓN
}

\author{
Nora Rodríguez García \\ Profesora ayudante de Derecho civil \\ Universidad de La Laguna
}

\section{RESUMEN}

Las plataformas de economía colaborativa juegan hoy un papel fundamental en el desarrollo de las relaciones comerciales digitales, y con el nacimiento de este modelo de intercambio se generan una serie de cuestiones jurídicas que el legislador nacional e internacional ha preferido abordar de manera cautelosa. Se estudia en este trabajo el debate en torno a la responsabilidad de los intermediarios a través del análisis de la Ley de Servicios de la Sociedad de la Información y la dicotomía entre el intermediario digital y el proveedor de servicios, analizando las dificultades existentes para su distinción y graduación de su régimen de responsabilidad. Con la vista en la propuesta de Reglamento de Servicios Digitales, la inexistencia de una legislación internacional vigente que abarque esta casuística implica que se deba resolver mediante normas de Derecho Internacional Privado para subsumirse en la legislación interna de cada país, aunque existen unos regímenes "generales» de responsabilidad contractual y extracontractual, los cuales serán objeto de estudio de este artículo.

Palabras Clave: economía colaborativa, responsabilidad civil, intermediarios digitales, proveedores de servicios.

\section{LIABILITY OF THE COLLABORATIVE ECONOMY PLATFORMS IN THE LIGHT OF LAW 34/2002 ON INFORMATION SOCIETY SERVICES}

\section{Abstract}

Collaborative economy platforms now play a fundamental role in the development of digital commercial relations and with the birth of this model of exchange, a series of legal questions have arisen that national and international legislators have preferred to approach cautiously. This paper examines the debate surrounding the liability of intermediaries through an analysis of the Law on Information Society Services and the dichotomy between the digital intermediary and the service provider, analysing the difficulties that exist in distinguishing between them and graduating their liability regime. With a view to the proposed Digital Services Regulation, the lack of current international legislation covering this casuistry means that it must be resolved by means of Private International Law rules to be subsumed in the domestic legislation of each country, although there are some "general" regimes of contractual and non-contractual liability, which will be the subject of study in this article. KEYWORDs: collaborative economy, liability, digital intermediaries, service providers. 
Las plataformas de economía colaborativa juegan hoy un papel fundamental en el desarrollo de las relaciones comerciales digitales, especialmente en lo que respecta al nexo en que las partes intervinientes son particulares o peers. El surgimiento de este modelo de intercambio plantea una serie de cuestiones jurídicas que tanto los legisladores nacionales como los internacionales han preferido abordar de manera cautelosa. Estamos ante un modelo de mercado muy reciente y fuertemente marcado por el nacimiento de internet en los ańos noventa del pasado siglo, pero con un desarrollo y crecimiento exponencial, lo cual hace muy difícil que se recoja adecuada y satisfactoriamente en la legislación actual. Ello es lo que explica que, a día de hoy, en España, la única normativa aplicable consiste en directivas de la UE transpuestas a las leyes españolas, pues tampoco contamos todavía con una legislación internacional aprobada que aborde este nuevo sistema en su conjunto.

La sentencia del TJUE de 13 de mayo de $2014^{1}$ sobre el derecho al olvido ha reabierto el debate en torno a la responsabilidad de los intermediarios, ya que la inexistencia de una legislación internacional que abarque la casuística que se viene produciendo implica que se deba resolver mediante normas de Derecho Internacional Privado para subsumirse en la legislación interna de cada país, aunque existen unos regímenes "generales» de responsabilidad contractual y extracontractual, de los que nos ocuparemos a lo largo del trabajo. En general, se tiene la idea de que esta solución sabe a poco, sobre todo debido al carácter internacional intrínseco de la economía colaborativa y a su desarrollo a través de internet. La globalización y la apertura del espacio digital, que rompe con las barreras geográficas y políticas, forman parte, pues, de su naturaleza, y se hace necesario darle soluciones jurídicas particularizadas.

En este trabajo se analiza un aspecto muy concreto de este fenómeno: la responsabilidad de las plataformas de economía colaborativa en el caso español cuando actúan, bien como intermediaria digital, o bien como proveedor del servicio; es decir, presuponiendo que las normas de conflicto nos hayan llevado a la designación de la legislación española como ley aplicable.

En primer lugar, haremos una breve introducción a la economía colaborativa, en la que esbozaremos los principales aspectos del tema que nos ocupa. Así, explicaremos los principios y elementos básicos que la conforman y su funcionamiento, para poder entender la responsabilidad de los intermediarios que la constituyen.

En segundo lugar, desarrollaremos el marco normativo analizando y explicando brevemente las directivas internacionales que se han dictado sobre la materia, así como su transposición a la legislación española. Con ello queremos conocer y diferenciar los regímenes existentes con respecto a la responsabilidad de las plataformas.

En tercer lugar, entrando propiamente en la materia, abordaremos las bases constitutivas y rasgos diferenciales de los dos tipos de plataformas digitales: la intermediaria digital y la proveedora de servicios.

En cuarto lugar, debido a que la implicación de ambas en el control de sus actividades influirá directamente en el tipo o grado de responsabilidad que, en caso

\footnotetext{
${ }^{1}$ Sentencia de 13 de mayo de 2014, C131/12, ECLI:EU:C:2014:317.
} 
de producir algún perjuicio, acarreará, centraremos el análisis en los artículos 13 a 17 de la LSSI. Debemos señalar que no nos centraremos en el tipo de ilícito cometido, como, por ejemplo, el derecho de libertad de expresión, el derecho de autor, el derecho al honor y la intimidad, etc., sino que estudiaremos los regímenes por los que se deberá regir su responsabilidad. No debemos olvidar que, una vez que se cometa el ilícito, deberemos atender también a las normas sectoriales existentes. Es por ello por lo que solo nos centraremos en el momento previo, es decir, en la situación en la que se estudia si un acto en una determinada plataforma genera responsabilidad para la misma.

Finalmente, en quinto lugar, expondremos algunas conclusiones extraídas del análisis de la materia objeto de estudio.

\section{INTRODUCCIÓN A LA ECONOMÍA COLABORATIVA}

La economía colaborativa es uno de los fenómenos jurídico-sociales más importantes de los últimos tiempos ${ }^{2}$. Se presenta como un nuevo modelo de prestación de servicios o bienes en el que se prioriza el acceso, que no la propiedad, sobre bienes o servicios y, de esta manera, el motor y el objeto principal de la economía colaborativa es compartir, intercambiar, distribuir, alquilar, etc. ${ }^{3}$. La cantidad de oferta y demanda a la que acceden las plataformas de economía colaborativa, debido al hecho de que al cambiar al intermediario tradicional por el intermediario digital se eliminan las barreras geográficas y se reducen los costes de transacción, hace que autores como Domenech $(2015)^{4}$ resalten que la crisis económica que se inicia en 2008 haya incentivado el florecimiento de la economía colaborativa, teniendo en cuenta que resulta muy atractivo para un ciudadano medio acceder a bienes y servicios de los que había quedado privado precisamente a consecuencia de esta crisis.

Para situar la materia, me valdré de la definición de economía colaborativa propuesta en la Comunicación de la Comisión al Parlamento Europeo titulada «Una Agenda Europea para la economía colaborativa» $(2016)^{5}$, que, a pesar de no ser de

2 En general, se considera que Rachel Botsman es la líder del movimiento, pues fue la que puso de relieve y en el punto de mira la importancia de la economía colaborativa en su obra What's mine is yours (Harper Collins, 2010).

${ }^{3}$ La Comisión Nacional de los Mercados y la Competencia (en adelante CNMC) la define en sus "Conclusiones preliminares sobre los nuevos modelos de prestación de servicios y la economía colaborativa» como el "fenómeno que se caracteriza como el mejor aprovechamiento de los recursos infrautilizados, mediante la puesta a disposición de los usuarios de las plataformas recursos, bienes o servicios». Disponible en https://docs.google.com/document/d/1n65MjUaTmRLuZCqTIlqyWvobVqreR-iAzsz1mhxy2y0/edit.

${ }^{4}$ Doménech Pascual, G. «La regulación de la economía colaborativa (El caso «Uber contra el taxi») Ceflegal». Revista práctica de Derecho, 2015, vol. 175-176, 61-104.

5 Comunicación de la Comisión al Parlamento Europeo, al Consejo, al Comité Económico y Social Europeo y al Comité de las Regiones: «Una Agenda Europea para la economía colabo- 
rabiosa actualidad, constituye uno de los primeros documentos europeos que realizan un esfuerzo de comprensión de este fenómeno. Así pues, a los efectos de lo que nos interesa en este trabajo, nos serviremos de la definición que se recoge en Una Agenda Europea (p. 3):

el término economía colaborativa se refiere a modelos de negocio en los que se facilitan actividades mediante plataformas colaborativas que crean un mercado abierto para el uso temporal de mercancías o servicios ofrecidos a menudo por particulares. La economía colaborativa implica a tres categorías de agentes i) prestadores de servicios que comparten activos, recursos, tiempo y/o competencias - pueden ser particulares que ofrecen servicios de manera ocasional («pares») o prestadores de servicios que actúan a título profesional ("prestadores de servicios profesionales»); II) usuarios de dichos servicios; III) intermediarios que -a través de una plataforma en línea- conectan a los prestadores con los usuarios y facilitan las transacciones entre ellos ("plataformas colaborativas»).

El nacimiento de la economía colaborativa pone, pues, sobre el plano jurídico una serie de nuevos e importantes retos: la redefinición de conceptos, deberes y obligaciones contraídos a través de canales no tradicionales, relaciones jurídicas generadas de forma atípica que hasta ahora el derecho no había tenido que regular, ya que no desarrollaban un papel subsumible en la normativa existente o, al menos, no habían generado controversia en cuanto a la resolución de conflictos. Todo ello nos lleva a una cuestión clave: ¿es necesaria la creación de nuevas normas jurídicas que cubran la casuística generada por la economía colaborativa? $\mathrm{O}$, por el contrario, ¿permite la regulación actual nacional e internacional dar respuesta a las situaciones controvertidas que esta nueva forma de acceder a la contratación genera?

Además, surgen nuevas figuras, como las plataformas de economía colaborativa, pilar básico de este fenómeno y a través de las cuales se realizan todas las transacciones que pueden ser consideradas de consumo colaborativo. Es una herramienta sin la cual no estaríamos dentro de este tipo de negocio jurídico. Estas son un instrumento tecnológico y digital que pone en contacto la oferta y la demanda, es decir, a los usuarios. $\mathrm{Y}$ en este contexto se pueden comportar de dos maneras, como intermediarios digitales y como proveedores de servicios. En esta contribución se analiza el primer caso, es decir, la responsabilidad de los intermediarios, cuya actividad fundamental es poner en contacto a los prestadores de servicios que desarrollen una actividad profesional o no profesional en el plano online con los usuarios que demanden el mismo.

Las relaciones jurídicas en la economía colaborativa tienen una estructura triangular, pues se dan relaciones simultáneas entre los tres tipos de agentes intervinientes: la PLATAFORMA (intermediario digital), el PRESTADOR DE SERVICIOS (profesional o particular que oferta un servicio a los usuarios que lo demanden: podrá ser

rativa». Publicado en el Registro de documentos de la Comisión el 2 de junio de 2016. Documento COM (2016)356/F1. En adelante, Una Agenda Europea. 
tanto la propia plataforma como el agente externo que hace uso de ella para desarrollar su actividad profesional) y el USUARIO (entendiendo como tal tanto el consumidor usuario del servicio como el profesional usuario de la plataforma). Es por ello por lo que determinar la responsabilidad de los intermediarios digitales es una tarea ardua, ya que, en esta encrucijada, estos muchas veces no actuarán solo como intermediarios, sino también como prestadores de servicios.

El problema principal que enfrenta la economía colaborativa es la inseguridad jurídica que se produce por la falta de conocimiento para aplicar los marcos jurídicos existentes; ello no significa que se desconozca lo permitido y lo prohibido, sino que una vez que se produce una lesión de derechos, un menoscabo de derechos, no resulta claro a dónde tenemos que acudir para subsanarlo. Tal y como señala la Comunicación en Una Agenda Europea, anteriormente citada, uno de los principales efectos de la economía colaborativa es que se desdibujan los límites establecidos entre pares como consumidor y proveedor, trabajador por cuenta propia y trabajador por cuenta ajena, etc. Este tipo de cuestiones son tratadas en las legislaciones propias de cada país y su diferencia cada vez es menos clara; de esta manera, los derechos y obligaciones de cada una de las partes varían, se transforman y se adaptan al caso concreto.

\section{MARCO NORMATIVO}

En este apartado nos ocuparemos del marco normativo que incide directamente o indirectamente con la responsabilidad. Lo haremos haciendo un repaso de la normativa nacional o internacional que se ha de tener en cuenta a la hora de establecer el régimen de responsabilidad por el que se regirán las plataformas. Para analizar el marco normativo existente se han de tener en cuenta las normas de Derecho Internacional Privado que determinarán la competencia judicial internacional y la ley aplicable. Sin embargo, el supuesto sobre el que se trabaja es que efectivamente conocerá de esta incidencia la normativa española ${ }^{6}$. Por otro lado, dejaremos de lado la normativa específica relativa al tipo de ilícito cometido y analizaremos las normativas que se pronuncian acerca de la responsabilidad de las plataformas.

Ante un sistema en el que se ha de atender caso por caso, ya que dependiendo de los factores que intervengan se abrirá la posibilidad de aplicar unas leyes u otras, lo lógico es abordar su estudio por orden cronológico; de este modo, se podrá comprobar si las distintas problemáticas que suscitaban las primeras legislaciones publicadas han ido siendo subsanadas a lo largo del tiempo ${ }^{7}$.

${ }^{6}$ Se ha de poner de relieve que, tratando el supuesto de hecho desde el inicio, en primer lugar deberíamos acudir al Reglamento Roma I, Reglamento (CE) n. ${ }^{\circ}$ 593/2008 del Parlamento Europeo y del Consejo, de 17 de junio de 2008, sobre la ley aplicable a las obligaciones contractuales, o al Reglamento Roma II, Reglamento (CE) n. ${ }^{\circ}$ 864/2007 del Parlamento Europeo y del Consejo de 11 de julio de 2007, relativo a la ley aplicable a las obligaciones extracontractuales.

7 En este punto es necesario reiterar la corta vida de este fenómeno, por lo que en el transcurso de este breve espacio de tiempo, no es común que se haya publicado normativa que supere e 
2.1. La Directiva 2000/31/CE del Parlamento Europeo y del Consejo, de 8 DE JUNIO DE 2000, Y SU INCORPORACIÓN AL ORDENAMIENTO JURÍDICO ESPAÑOL por la Ley 34/2002 de Servicios de la Sociedad de la Información y de Comercio Electrónico

Todo comienza con la Directiva 2000/31/CE del Parlamento Europeo y del Consejo, de 8 de junio de 2000, relativa a determinados aspectos jurídicos de los Servicios de la Sociedad de la Información, en particular el comercio electrónico en el mercado interior (Directiva sobre el comercio electrónico, en adelante DCE). Propuesta por la Comisión y en virtud del Tratado constitutivo de la Unión Europea, el Parlamento Europeo adopta esta Directiva, reforzando así la idea de la existencia de un único mercado interior europeo sin fronteras ni barreras y garantizando la libre circulación de los servicios de la sociedad de la información en los Estados Miembros. Esta directiva, posteriormente transpuesta por la Ley 34/2002 de Servicios de la Sociedad de la Información y de Comercio Electrónico, se configura como necesaria y susceptible de ser superada en nivel de protección por los Estados Miembros y ha servido para definir nuevos conceptos antes no regulados: servicios de la sociedad de información, prestador de servicios, prestador de servicios definido, destinatario, comunicación, etc.; y también para establecer los principios y requisitos básicos por los que se ha de regir un agente o prestador de servicios para desarrollar su actividad y fomentar la idea de desarrollo del mercado interior.

Antes de analizar en profundidad la Ley 34/2002 de Servicios de la Sociedad de la Información y de Comercio Electrónico (en adelante LSSI), debemos evidenciar que, tal y como señala Montero Pascual $(2017)^{8}$, el iter legislativo con respecto a la conceptualización de SERVICIO DE LA SOCIEDAD DE LA INFORMACIÓN COMO categoría jurídica comienza con la Directiva 98/48/CE 9 , que, a su vez, modifica la Directiva 98/34/CE ${ }^{10}$, calificándolo en su artículo 2 como «todo servicio prestado normalmente a cambio de una remuneración, a distancia, por vía electrónica y a petición individual de un destinatario de servicios. A efectos de la presente definición, se entenderá por "a distancia", un servicio prestado sin que las partes estén presentes simultáneamente; "por vía electrónica", un servicio enviado desde la fuente y recibido por el destinatario mediante equipos electrónicos de tratamiento (incluida la compresión digital) y de almacenamiento de datos y que se transmite, canaliza y recibe enteramente por hilos, radio, medios ópticos o cualquier otro medio electro-

introduzca cambios reales como consecuencia de la problemática existente.

${ }^{8}$ Montero Pascual, J.J. La regulación de la economía colaborativa: Airbnb, BlaBlaCar, Uber y otras plataformas. Tirant lo Blanch, Valencia. 2017.

9 Directiva 98/48/CE del Parlamento Europeo y del Consejo de 20 de julio de 1998, que modifica la Directiva 98/34/CE, por la que se establece un procedimiento de información en materia de las normas y reglamentaciones técnicas, DOCE L 217/18, de 5.8.1998.

${ }_{10}$ Directiva 98/34/CE del Parlamento Europeo y del Consejo, de 22 de junio de 1998, por la que se establece un procedimiento de información en materia de las normas y reglamentaciones técnicas, DOCE 204/37, de 21.7.1998. 
magnético; y por "a petición individual de un destinatario de servicios", un servicio prestado mediante transmisión de datos a petición individual».

La LSSI en su exposición de motivos establece que «se acoge, en la Ley, un concepto amplio de "servicios de la sociedad de la información", que engloba, además de la contratación de bienes y servicios por vía electrónica, el suministro de información por dicho medio (como el que efectúan los periódicos o revistas que pueden encontrarse en la red), las actividades de intermediación relativas a la provisión de acceso a la red, a la transmisión de datos por redes de telecomunicaciones, a la realización de copia temporal de las páginas de Internet solicitadas por los usuarios, al alojamiento en los propios servidores de información, servicios o aplicaciones facilitados por otros o a la provisión de instrumentos de búsqueda o de enlaces a otros sitios de Internet, así como cualquier otro servicio que se preste a petición individual de los usuarios (descarga de archivos de vídeo o audio...), siempre que represente una actividad económica para el prestador». En relación con esto, debemos considerar lo seńalado por Rodríguez de las Heras Ballell (2011), que entiende que se comprenden dentro de esta Ley los servicios no remunerados ${ }^{11}$, siempre que constituyan una actividad económica para el prestador, lo que nos permite un mayor rango de aplicación de la misma ${ }^{12}$. Así, el objetivo de la Ley es claramente la regulación del régimen jurídico de los servicios de la sociedad de la información y la contratación electrónica. Lo que interesa para el análisis de esta Ley es el régimen de responsabilidad que se establece en los artículos 13-17, instituyendo en la misma cinco tipos de prestadores de servicio, subsumibles en cada uno de los artículos, que guían el régimen por el que se han de regir. De esta manera, distingue entre:

a) Responsabilidad de los prestadores de los servicios de la sociedad de la información.

b) Responsabilidad de los operadores de redes y proveedores de acceso.

c) Responsabilidad de los prestadores de servicios que realizan copia temporal de los datos solicitados por los usuarios.

d) Responsabilidad de los prestadores de servicios de alojamiento o almacenamiento de datos.

e) Responsabilidad de los prestadores de servicios que faciliten enlaces a contenidos o instrumentos de búsqueda.

Esta Ley no establece cuál es el régimen por el que se debe regir la responsabilidad de las plataformas, sino cuáles son las exenciones a la responsabilidad.

${ }^{11}$ El prisma de esta afirmación reside en que no ha de ser el destinatario del mismo (el consumidor) el que abone el precio del servicio aun obteniéndolo, como puede ser en el caso de la publicidad y comunicaciones comerciales: no es un servicio remunerado por el consumidor y constituye parte de la actividad económica de la plataforma.

12 Rodríguez de las Heras Ballell, T., «Régimen jurídico de los acuerdos clickwrap y browsewrap». Revista Iberoamericana de Derecho de Autor, 2011-año v, (9), 82-113. 


\subsection{Directiva 2011/83/UE del Parlamento Europeo y del Consejo, de 25 de OCTUBRE DE 2011, SOBRE LOS DERECHOS DE LOS CONSUMIDORES}

Por último, nos encontramos ante la Directiva 2011/83/UE del Parlamento Europeo y del Consejo, de 25 de octubre de 2011, sobre los derechos de los consumidores. El problema que presenta la aplicación de esta directiva a la materia es el ámbito mismo de aplicación de la directiva especifica, que solo será aplicable a los contratos celebrados entre un comerciante y un consumidor; sin embargo, esto no se refleja de manera acertada en la naturaleza de las plataformas de economía colaborativa, ya que pueden ser $\mathrm{C} 2 \mathrm{C} / \mathrm{P} 2 \mathrm{P}, \mathrm{B} 2 \mathrm{~B}$ o $\mathrm{B} 2 \mathrm{C}$ (como vemos, solo aplicaría en el último caso):

a) $\mathrm{C} 2 \mathrm{C} / \mathrm{P} 2 \mathrm{P}$ (consumer to consumer/peer to peer): entre particulares que ejercen de manera puntual el ofrecimiento de servicios a la sociedad de la información.

b) B2B (business to business): entre comerciantes.

c) B2C (business to consumer): entre comerciantes y consumidores.

Aunque por su naturaleza las plataformas de la economía colaborativa están destinadas a tener un modelo $\mathrm{C} 2 \mathrm{C}$, es inevitable que en determinadas plataformas o aplicaciones ${ }^{13}$ exista una tendencia que incluye cada vez más sujetos profesionales o semiprofesionales de la materia ${ }^{14}$, lo que la convierte en una plataforma en la que también se pueden dar relaciones B2C. De manera generalizada, en las plataformas coexisten relaciones $\mathrm{C} 2 \mathrm{C}$ y $\mathrm{B} 2 \mathrm{C}$ sin que haya especial fricción, aunque una de las cuestiones más debatidas acerca de la responsabilidad es si la plataforma debiera distinguir entre profesionales y no profesionales. Recordemos que en la Comunicación de la Comisión Una Agenda Europea se indica que en la legislación de la Unión Europea no se establece expresamente cuándo, en qué momento y bajo qué circunstancias un peer se convierte en un prestador de servicios profesional. En este caso, se ha de estar a los umbrales que establece la legislación interna de cada país.

Como solución a ello, esta Directiva incorpora en su considerando 13 que «un Estado Miembro podrá mantener o introducir normas de Derecho interno que correspondan a las disposiciones de la presente Directiva o a algunas de las disposiciones de la misma respecto de contratos que queden fuera del ámbito de aplicación

${ }_{13}$ Se entiende aquí por aplicaciones lo que recoge el Diccionario de la lengua española de la RAE en la acepción cuarta de la definición de esta palabra: «Inform. Programa preparado para una utilización específica, como el pago de nóminas, el tratamiento de textos, etc.». Disponible en https:// dle.rae.es/aplicación?m=form.

${ }^{14}$ Un ejemplo de ello es la plataforma Airbnb, destinada al alquiler (por días, semanas o meses) de una vivienda que, generalmente, constituye la segunda residencia de los propietarios o bien otra de sus propiedades; en teoría, Airbnb está destinada a consumidores (que, por tanto, no ejercen actividad profesional), quienes establecen una relación que les facilita liberarse de las cargas que puede generar la propiedad y les permite aprovechar bienes que están infrautilizados; en la práctica, cada vez más agentes de la propiedad o inmobiliarios usan esta aplicación para ejercer su actividad profesional. 
de la presente Directiva», entendiendo que los derechos que afectan a los consumidores de los servicios de la sociedad de la información deberán quedar sujetos a esta Directiva, a pesar de no encontrarse en su ámbito de aplicación. Se trata de una Directiva que incorpora derechos para los consumidores de importancia vital, tal y como el derecho de información y desistimiento en los contratos celebrados a distancia o fuera del establecimiento, ámbito en el que se desarrolla la actividad principal de los prestadores de servicios de la sociedad de la información.

Una vez expuesto brevemente el marco jurídico existente hasta ahora, se observan dos aspectos: uno, que la normativa que lo regula no es reciente; y dos, que el crecimiento exponencial de la tecnología (factor determinante de este fenómeno) hace que los presupuestos que se contemplaban hace quince años y que eran susceptibles de regulación han quedado desfasados. Actualmente, y cada vez más, las plataformas de economía colaborativa se comportan tanto como intermediarios como como prestadores de servicios. El hecho de que la normativa existente solo regule la responsabilidad de los intermediarios deriva en que no exista una respuesta homogénea en cuanto a la responsabilidad total. En el momento en el que se introdujo la LSSI, la actividad principal de las plataformas era como intermediarios digitales; tenemos que pensar en herramientas como Google y en la inexistencia de negocios tales como Facebook o Airbnb, que son ejemplos de economía colaborativa recientes y de mayor amplitud, en los que podemos observar cómo sus actividades exceden del comportamiento de intermediario digital puro y pasan a prestar un servicio de la sociedad de la información. Es por ello por lo que, superados actualmente los presupuestos por los cuales se dictaron estas normativas, se hace necesaria una revisión de las mismas.

2.3. Directiva (UE) 2019/770 del Parlamento Europeo y del Consejo, de 20 DE MAYO DE 2019, RELATIVA A DETERMINADOS ASPECTOS DE LOS CONTRATOS DE SUMINISTRO DE CONTENIDOS Y SERVICIOS DIGITALES

De reciente adopción, y aportando mayor luz sobre la materia, la Directiva (UE) 2019/770 del Parlamento Europeo y del Consejo, de 20 de mayo de 2019, relativa a determinados aspectos de los contratos de suministro de contenidos y servicios digitales ${ }^{15}$, introduce en su considerando 18 una nueva vía por la cual los Estados Miembros disponen de la libertad de calificar a los prestadores de servicios como empresarios cuando estos no actúen en «calidad de socio contractual directo del consumidor", pudiendo entonces ser responsables por la no conformidad del suministro de contenidos o servicios digitales a los consumidores, quedando sujetos a

${ }_{15}$ El corpus de esta Directiva contiene, tal y como expresa su artículo 1, «normativa relativa a la conformidad de los contenidos o servicios digitales en el contrato, las medidas correctoras en caso de falta de conformidad o incumplimiento del suministro y las modalidades para exigirlas, y la modificación de los contenidos o servicios digitales». 
la «Responsabilidad del empresario» determinada en el artículo 11 de la Directiva, así como los artículos 12-16 relativos a la carga de la prueba, medidas correctoras por incumplimiento, medidas correctoras por falta de conformidad y ejercicio del derecho a la resolución del contrato, respectivamente. Se establece así una vía de resolución y régimen de responsabilidad que permite una observancia del problema sin la delimitación del caso por caso, previo a la atención de las normativas sectoriales, debido a que el grado de armonización de esta Directiva es pleno, reforzando la seguridad jurídica para consumidores y empresarios.

\subsection{La propuesta de Reglamento de Servicios Digitales (Digital Services Act)}

Por último, y enfocando la vista hacia el futuro, no podemos dejar de mencionar la propuesta de Reglamento de Servicios Digitales (Digital Services Act, en adelante DSA) publicada el 15 de diciembre de $2020^{16}$ por la Comisión Europea, perteneciente al paquete de medidas que tomó forma a raíz de la estrategia Shaping Europe's Digital Future ${ }^{17}$. Esta propuesta de Reglamento tiene como objetivo crear un espacio digital que goce de mayor seguridad jurídica, protegiendo los derechos fundamentales de los usuarios de servicios digitales. En la misma, se contempla una nueva batería de derechos y obligaciones para los proveedores de servicios de intermediación, los proveedores de servicios relativos al alojamiento de datos, las plataformas online y las plataformas online de gran tamaño ${ }^{18}$. De esta manera, la DSA se propone mejorar significativamente los mecanismos de eliminación de contenido ilegal para la efectiva protección de los derechos fundamentales de los usuarios, incluyendo el derecho a la libertad de expresión, a través de siete medidas: a) establecimiento de mecanismos de detección de contenido, servicios o bienes ilícitos por parte de los usuarios o los trusted flaggers (alertadores fiables) ${ }^{19}$, b) nuevas obligaciones sobre trazabilidad de los usuarios comerciales para ayudar a la detección de posibles vendedores de productos ilegales, c) nuevas garantías para los usuarios ${ }^{20}$,

16 Disponible para su consulta en https://eur-lex.europa.eu/legal-content/es/TXT/?qi$\mathrm{d}=1608117147218 \&$ uri $=$ COM\%3A2020\%3A825\%3AFIN.

${ }^{17}$ Esta estrategia se basa en cuatro pilares fundamentales: tecnología al servicio de la sociedad, un entorno de competencia digital económica justa, una sociedad digital sostenible, democrática y abierta y la concepción de Europa como actor digital mundial.

${ }_{18}$ El considerando 54 de la propuesta establece que las plataformas de muy gran tamaño entrańan un riesgo para la sociedad una vez que el número de destinatarios de una plataforma asciende a un porcentaje importante de la población de la Unión que se fija en el 10\% de la población de la Unión, umbral que se deberá mantener actualizado.

19 Nueva figura que introduce la DSA, que permite que una entidad habilitada como tal pueda informar de manera prioritaria a la plataforma sobre el contenido ilícito que la misma aloja, transmite, facilita, etc.

${ }^{20}$ Como, por ejemplo, la posibilidad de impugnar decisiones de moderación de contenidos de las plataformas. 
d) medidas adicionales de transparencia para las plataformas digitales ${ }^{21}$, e) obligaciones para las plataformas muy grandes para evitar abusos en sus sistemas, $\mathrm{f}$ ) acceso por parte de investigadores a datos claves de estas plataformas muy grandes para analizar la evolución de los riesgos online y g) una nueva estructura de supervisión de las plataformas muy grandes adecuada a la actual complejidad del ciberespacio ${ }^{22}$.

\section{LAS PLATAFORMAS DE ECONOMÍA COLABORATIVA. DICOTOMÍA ENTRE EL INTERMEDIARIO DIGITAL Y EL PROVEEDOR DE SERVICIOS}

Las plataformas de economía colaborativa tienen una función que ha sido determinada; nos valemos de nuevo de la definición que establece la Comunicación de la Comisión Una Agenda Europea, que las define como entes que proporcionan un «servicio prestado normalmente a cambio de una remuneración, a distancia, por vía electrónica y a petición individual de un prestatario de servicios, ofrecen un servicio de la sociedad de información ${ }^{23}$. La estructura tripartita de relaciones entre las partes intervinientes, a la que hemos hecho referencia más arriba, hace que conceptos básicos del derecho, como pueden ser consumidor, profesional, proveedor, prestador de servicios, etc., se actualicen, alteren y evolucionen, ya que, como se ha reiterado, sus características principales han ido cambiando. Del mismo modo en que las figuras del profesional y el consumidor se han ido confundiendo, lo cual ha derivado en un nuevo concepto y nueva figura, el prosumer ${ }^{24}$, las figuras de interme-

${ }^{21}$ Contempladas en la propuesta en su Capítulo III, "Obligaciones de diligencia debida para crear un entorno en línea transparente y seguro", estas medidas adicionales hacen referencia a un amplio rango de actuaciones que comprenden desde la emisión de informes por parte de las plataformas digitales de intermediación que contengan información sobre cualquier actividad de moderación de contenidos (incluyendo número de órdenes recibidas por autoridades de los Estados, número de avisos, actividad de moderación realizada por iniciativa propia y número de reclamaciones recibidas), mecanismos de notificación y acción para prestadores de servicios de alojamiento de datos, hasta el envío de una exposición de motivos al destinatario del servicio cuando se retiren elementos de información o se inhabilite el acceso a la misma, entre otros.

${ }^{22}$ Gracias a la creación de un Consejo Europeo de Servicios Digitales.

${ }^{23}$ Definición extraída del art. 2 a) de la Directiva 2000/31/CE.

${ }^{24}$ Concepto que surge para aquellos particulares que actúan como profesionales en un determinado negocio jurídico; por ejemplo, el particular que alquila periódicamente su vivienda a terceros puede ser considerado un prosumer, ya que tiene una cierta experiencia en el tipo de negocio, y además se beneficia de él. No constituye su medio de vida, pero sí le aporta una retribución. Se trata de un particular que está más próximo a nuestro concepto de consumidor que al de profesional. Las cargas, responsabilidades y obligaciones de un profesional, a las que se añade la necesidad de observancia de la legislación que le atańe, resultarían arduas para un peer, que no desempeña esta labor en términos de actividad profesional. Para ahondar sobre este término se recomienda la lectura de Sánchez Carrero, J. y Contreras Pulido, P., «De cara al prosumidor: producción y consumo empoderando a la ciudadanía 3.0». ICONO 14, vol. 10, n. ${ }^{\circ} 3,62-84$. 2012; JARNE Muñoz, P.: «El prosumidor como figura clave en el desarrollo del Derecho de consumo derivado del mercado 
diario digital y proveedor de servicios han terminado fundiéndose, aunque aún no existe un término que lo conceptualice. Pero lo que es cierto es que muchas veces, y cada vez más, estamos ante plataformas (es decir, el instrumento mediante el cual se relacionan las partes) que pueden actuar tanto como intermediario como como prestador de un servicio. E identificar cada situación es de vital importancia para la determinación de la responsabilidad civil de las plataformas, ya que se aplicarían normas distintas para determinarla.

Por tanto, ¿qué sucede cuando una plataforma actúa como un simple intermediario?: aplicamos la LSSI. ¿Qué sucede cuando actúa como un prestador de servicios?: aplicamos las normas estatales sobre responsabilidad contractual y extracontractual, atendiendo a las posibles normas sectoriales que puedan existir (como en el mercado del transporte o del alojamiento vacacional, por ejemplo). Pero ¿qué sucede cuando actúa como ambas?: en este caso, deberemos estudiar cuál es el nivel de implicación de la plataforma (sea prestadora o intermediaria) en el control de los contenidos que aloja, transmite, reproduce, guarda o redirecciona.

Hasta aquí, la definición de un intermediario digital o plataforma de economía colaborativa no parece presentar mayor complejidad; sin embargo, cuando dichas plataformas comienzan a ofrecer el servicio subyacente, es decir, aquel que los prestadores de servicios (ya sean $\mathrm{C} 2 \mathrm{C}$ o $\mathrm{B} 2 \mathrm{C}$ ) proporcionan, la responsabilidad de las mismas se verá incrementada y será difícil de atribuir. Con los presupuestos que contempla la Ley podemos determinarla claramente cuando actúan como una y como otra, pero, cuando están a caballo entre ambas actividades, deberemos atender a otro tipo de consideraciones, de las que nos ocuparemos a continuación.

Existen tres sistemas generales de régimen de responsabilidad ${ }^{25} \mathrm{y}$ dentro de ellos se podrán subsumir los casos diferenciados de intermediarios digitales y prestadores de servicios:

A. Exención absoluta de responsabilidad: se da inmunidad prácticamente absoluta sobre el contenido recabado en las plataformas, siempre que no intervenga sobre el mismo y siempre que, cuando reciba orden de eliminarlo, colabore con la autoridad competente (régimen presente en Chile, Estados Unidos, etc.).

B. Responsabilidad objetiva: se requiere a los intermediarios que monitoreen activamente el contenido de las plataformas, ya que de lo contrario se podrán enfrentar a acciones legales (régimen presente en China).

C. 'Safe harbors' o puertos seguros: los intermediarios serán eximidos de toda responsabilidad siempre que cumplan con los requisitos de notificación y retirada. La notificación y retirada se produce cuando los usuarios alertan a

digital». Revista CESCO de Derecho de Consumo, n. ${ }^{\circ}$ 19/2016, 41-51,2012; y Jarne Muñoz, P. Economía colaborativa y plataformas digitales. Editorial Reus, Madrid, 2020.

25 Tomado de Article 19: «Los intermediarios del Internet: Dilema de responsabilidad -sesión de preguntas y respuestas». Agosto de 2013. Disponible en https://www.article19.org/es/ resources/internet-intermediaries-dilemma-liability-q/. 
la plataforma de la existencia de un contenido presuntamente ilegal, y en caso de que no eliminen el contenido de la plataforma podrán estar sujetos a responsabilidad (régimen presente en Singapur, Ghana, Uganda y la UE).

Pese a que la LSSI habla de cuatro tipos de «agentes» de la sociedad de la información, realmente estos agentes solo hacen referencia a cuatro tipos de intermediarios digitales: operadores de redes y proveedores de acceso; aquellos que realizan copia temporal de los datos solicitados por los usuarios; prestadores de servicios de alojamiento o almacenamiento de datos; y aquellos que facilitan enlaces a contenidos o instrumentos de búsqueda. De la letra de la Ley no se desprende con claridad si se trata de intermediarios digitales puros (aquellos que no prestan el servicio subyacente) o si se trata de prestadores de servicios puros (aquellos que sí lo prestan) hasta que habla del régimen de exoneración de la responsabilidad; es ahí cuando nos damos cuenta de que en el art. 13 de la LSSI se hace referencia a los prestadores de servicios (que no intermediarios digitales) y solo para dejar claro que se regirán por las normativas nacionales en términos de responsabilidad; mientras que para los intermediarios digitales detalla lo que antes denominábamos exoneración de la responsabilidad.

\section{LA RESPONSABILIDAD DE LOS INTERMEDIARIOS DIGITALES Y DE LOS PROVEEDORES DE SERVICIOS}

Para dilucidar la responsabilidad de los intermediarios digitales nos basaremos en la LSSI, ya que esta es la que establece el régimen de responsabilidad, o más precisamente, el régimen de exención de responsabilidad de los intermediarios digitales.

\subsection{Algunas notas previas}

Con respecto a los intermediarios digitales, partimos de la base de la exención absoluta, aunque para que se puedan "activar» estas exoneraciones siempre se debe cumplir con los presupuestos que se contemplan. La LSSI no contiene ninguna norma que afecte directamente a la responsabilidad civil de los proveedores de información: solo se ocupa de los prestadores intermediarios, es decir, de aquellos que no crean la información, sino que se limitan a permitir el acceso o difusión de la misma, diferencia clave que permite la delimitación de la responsabilidad. En el momento en el que la plataforma es creadora del contenido que se difunde o publica, siguiendo las reglas establecidas hasta ahora, será responsable de la ilegalidad del contenido que dicha información contenga. Sin embargo, tal y como apunta Cavanillas Múgica $(2007)^{26}$, que no se nombre en la LSSI a los proveedores de infor-

26 Cavanillas Múgica, Santiago, «La responsabilidad de los proveedores de información en la Ley 34/2002, de Servicios de la Sociedad de la Información y de Comercio Electrónico», en Responsabilidades de los proveedores de información en internet, 2007, pp. 1-39. 
mación en cuanto a la responsabilidad directa que puedan generar no significa que la responsabilidad de estos prestadores no pueda verse afectada indirectamente por la LSSI dada la existencia de lo que denomina como "casos problemáticos»" Para estos casos intermedios, estudia la relación entre la iniciativa del destinatario del servicio, la incorporación de la información mediante enlaces directos, el alojamiento de dichos datos recopilados por el prestador, la pasividad técnica del prestador y los datos proporcionados por el destinatario del servicio. Así mismo, en la obra Grimalt Servera $(2007)^{28}$ analiza otro posible "caso problemático» como es la prensa digital en relación con los servicios de intermediación y la afección indirecta la LSSI con respecto a la Ley 14/1966, de Prensa e Imprenta, con las complicaciones que surgen de la determinación del servicio de prensa digital como servicio de la sociedad de la información.

\subsection{LA RESPONSABILIDAD EN LA LSSI}

Como intermediarios digitales entendemos los operadores de redes y proveedores de acceso, los prestadores de servicios que realizan copia temporal de los datos solicitados por los usuarios, los prestadores de servicios de alojamiento o almacenamiento de datos y los prestadores de servicios que faciliten enlaces a contenidos o instrumentos de búsqueda. Así, los artículos 13-17 de la LSSI contemplan lo siguiente en cuanto a la responsabilidad:

1. Prestadores de servicios de acceso a la Red: dirigido a «operadores de redes de telecomunicaciones y proveedores de acceso a una red de telecomunicaciones que presten un servicio de intermediación que consista en transmitir por una red de telecomunicaciones datos facilitados por el destinatario del servicio o facilitar el acceso a esta. No serán responsables por la información transmitida salvo que ellos mismos hayan originado la transmisión, modificado los datos o seleccionado estos a los destinatarios de dichos datos». Por lo tanto, no serán responsables por el contenido que alojen pero sí tienen un deber de colaboración para/con las autoridades competentes cuando se les requiera interrumpir un servicio o retirar un contenido de la web.

2. Prestadores de servicio de copia temporal: «prestadores de un servicio de intermediación que transmitan por una red de telecomunicaciones datos facilitados por el destinatario del servicio y, con la única finalidad de hacer mucho más

27 Hace referencia a «terceros que hacen llegar una información, por vía electrónica o no, para que la aloje», «autor de una página web que coloca en la misma información tomada de otra fuente, con autorización o no y con cita o no de la fuente empleada" y "prestador de un servicio de foto o blog que se reserva expresamente el ejercicio de una función censora de contenidos ilícitos o incorrectos o que suspende la publicación de mensajes de terceros hasta que han pasado dicho filtro».

${ }^{28}$ Grimalt Servera, Pedro, «La responsabilidad de los proveedores de información en Internet y la Ley 14/1966, de Prensa e Imprenta», en la misma obra, pp. 41-81. 
eficaz su transmisión, almacenan una copia temporal» (memoria tampón o caching); no serán responsables del contenido de esos datos ni de la reproducción temporal siempre que se cumplan las siguientes condiciones, que transcribimos a continuación:

a) No modifican la información.

b) Permiten el acceso a ella solo a los destinatarios que cumplan las condiciones impuestas a tal fin, por el destinatario cuya información se solicita.

c) Respetan las normas generalmente aceptadas y aplicadas por el sector para la actualización de la información.

d) No interfieren en la utilización lícita de la tecnología generalmente aceptada y empleada por el sector, con el fin de obtener datos sobre la utilización de la información.

e) Retiran la información que hayan almacenado o hacen imposible el acceso a ella, en cuanto tengan conocimiento efectivo de:

1. ${ }^{\circ}$ Que ha sido retirada del lugar de la red en que se encontraba inicialmente,

2. ${ }^{\circ}$ que se ha imposibilitado el acceso a ella, o

$3 .^{\circ}$ que un tribunal u órgano administrativo competente ha ordenado retirarla o impedir que se acceda a ella.

3. Prestadores de servicio de alojamiento de datos: prestadores de un servicio de intermediación consistente en albergar datos proporcionados por el destinatario de ese servicio. En este caso, la letra de la Ley no especifica mucho más, por lo que nos encontramos ante la problemática encrucijada de definir exactamente qué es. La misma hace referencia a las actividades de housing y hosting, que las practica cualquier espacio en que se permita a usuarios distintos del administrador incorporar contenidos (por ejemplo: en los foros online se puede publicar una opinión y también facilitar el acceso a archivos). En este caso tampoco serán responsables por la información almacenada a petición del destinatario, siempre que, por una parte, no tengan conocimiento efectivo de que la actividad o la información almacenada es ilícita o de que lesiona bienes o derechos de un tercero susceptibles de indemnización; y, por otra, que si lo tienen, actúen con diligencia para retirar los datos o hacer imposible el acceso a ellos.

Como se observa, de nuevo se establece una presunción de «conocimiento efectivo" condicionada por los siguientes aspectos: a) Un órgano competente debe haber declarado la ilicitud de los datos, ordenado su retirada o que se imposibilite el acceso a los mismos, o se hubiera declarado la existencia de la lesión de bienes o derechos de un tercero susceptibles de indemnización; y b) Que el prestador conociera la correspondiente resolución, sin perjuicio de los procedimientos de detección y retirada de contenidos que los prestadores apliquen en virtud de acuerdos voluntarios y de otros medios de conocimiento efectivo que pudieran establecerse. 
Arroyo $(2020)^{29}$ propone una matización en el análisis y aplicación de la exención de responsabilidad en el caso de los prestadores de servicios de alojamiento de datos, abandonando la clasificación de los mismos según su rol activo o pasivo y reemplazando la misma por la apreciación del «grado de control», «desempeño de funciones editoriales» o "conocimiento efectivo», de los que hablaremos más adelante.

4. Prestadores de enlaces e instrumentos de búsqueda: «facilitan enlaces a otros contenidos o incluyen en los suyos directorios o instrumentos de búsqueda de contenidos». Al contrario que en los casos anteriores, en este punto el legislador español se desvió de lo dispuesto en la directiva comunitaria, que contemplaba la no responsabilidad de este tipo de prestadores de servicios, a la espera de un informe para analizar la necesidad de presentar responsabilidad de los proveedores de hipervínculos e instrumentos de localización. El legislador español, en vez de esperar al informe, utiliza el mismo método que en los artículos anteriores y exime de todo tipo de responsabilidad, siempre que estos «no tengan conocimiento efectivo de la actividad o la información a la que remiten o recomiendan es ilícita, o que pueden lesionar bienes o derechos de un tercero susceptibles de indemnización». Como conocimiento efectivo entiende «la existencia de una resolución de un órgano competente declarando, bien la ilicitud de los datos, ordenando su retirada o que se imposibilite el acceso a los mismos, bien la existencia de la lesión y, en segundo lugar, que el prestador de los servicios conozca esa resolución».

El principal escollo que presenta la aplicación de estos preceptos es la determinación del "conocimiento efectivo", pues no existe una homogeneidad de criterios para resolver si efectivamente se cumple este requisito o no de manera efectiva. En todos los casos analizados, un presupuesto objetivo de esta exención es que haya sido decretada por un órgano o autoridad competente, además de «otros medios de conocimiento efectivo que pudieran establecerse»; sin embargo, esto resulta contradictorio con el principio de notificación y retirada, ya que los propios usuarios podrían haber advertido del contenido ilícito o lesivo presente en la plataforma y esta obviarlo debido a que no proviene de una autoridad competente. En la propuesta de DSA elaborada por la Comisión Europea, se contempla la regulación del estatus de los alertadores fiables o trusted flaggers para que, evitando las posibles situaciones de abuso y menoscabo de derechos fundamentales como la libertad de expresión o el propio derecho a la información, las empresas de gran tamaño ${ }^{30}$ prioricen la retirada de contenido ilícito o el acceso al mismo a través de estas entidades habilitadas, sin perjuicio de las posibles notificaciones que puedan recibir por

29 Arroyo Amayuelas, E., «La responsabilidad de los intermediarios en internet: ¿Puertos seguros a prueba de futuro?». Cuadernos de Derecho Transnacional, 2020, n. . 1, pp. 808-837.

30 Se entienden por estas aquellas con un alcance del $10 \%$ de los 450 millones de usuarios que se estiman en Europa. 
parte de los usuarios individuales que así lo detecten ${ }^{31}$. Cabe la posibilidad de que se den situaciones en las que la plataforma conocía de manera efectiva la presencia de contenido ilícito y obvia su obligación de retirarlo, ya que sigue amparada en las exenciones de responsabilidad al no ser notificada por una autoridad competente, a la espera de una mayor aclaración por parte de la DSA. Al respecto de la LSSI, Peguera Ponch (2007) ${ }^{32}$ llama la atención sobre el hecho de que la jurisprudencia tampoco ha sido uniforme en la interpretación de estos artículos (art. 13-17), que varían desde una interpretación restrictiva (exigiendo la notificación por medio de resolución judicial o administrativa de la existencia de contenido ilícito) a una más amplia (entendiendo que el conocimiento efectivo de contenido ilícito se puede obtener por otros medios $\left.{ }^{33}\right)$.

Juliá-Barceló $(1998)^{34}$, anticipándose con su publicación a la estrategia que se propone actualmente con la DSA, se pronuncia acerca de la falta de regulación del sistema de notificación y retirada, y señala que es necesaria la «creación de un cuerpo especial, cuya composición habría de ser cuidadosamente elegida, encargado de recibir todas las notificaciones y decidir en qué casos debe actuar el proveedor de servicios», concepto que casa con el anteriormente denominado como trusted flaggers. De esta manera, «se tendría al intermediario por notificado, quedando obligado a retirar o bloquear el acceso al material presuntamente ilícito"; sin embargo, el texto actual de la propuesta DSA se aleja de esta imposición para todos los intermediarios digitales, entendiendo que esto puede ser dificultoso (y oneroso) para el intermediario digital medio (que no de gran tamaño), añadiendo una obligación adicional a cumplimentar para poder acogerse a los safe harbors, acreditando que disponen o colaboran con un cuerpo especial para el estudio de las notificaciones por hechos ilícitos.

31 Teniendo en cuenta que se puede dar el caso de repetidas notificaciones injustificadas producidas por usuarios que notifiquen contenidos lícitos de forma abusiva, afectando a los derechos de libertad de expresión o información de otros usuarios.

32 Peguera Ponch, M., (2007): «Sólo sé que no sé nada (efectivamente)»: la apreciación del conocimiento efectivo y otros problemas en la aplicación judicial de la LSSI. Monográfico del «III Congreso Internet, Derecho y Política (IDP). Nuevas perspectivas». Revista de Internet, politica $y$ derecho n. ${ }^{\circ}$ 5. 2007, pp. 2-18.

${ }^{33}$ Parecería que se produce aquí una alteración de la carga de la prueba; sin embargo, atendiendo a la Directiva (UE) 2019/770, relativa a determinados aspectos de los contratos de suministro de contenidos y servicios digitales, su considerando 59 nos indica que «si bien corresponde al consumidor aportar pruebas de que los contenidos o servicios digitales no son conformes, el consumidor no ha de tener que demostrar que la falta de conformidad existía en el momento del suministro de los contenidos o servicios digitales o, en caso de suministro continuo, durante la vigencia del contrato. En su lugar, debe ser el empresario quien demuestre la conformidad de los contenidos o servicios digitales en ese momento o durante ese periodo».

34 Juliá-Barceló, R., «Liability of online intermediaries: An european perspective». European Intellectual Property Review, 1998-Issue 12, pp. 1-9. 
Vistos los presupuestos que determina la Ley, Peguera Ponch $(2001)^{35}$ advierte que en esta directiva se establecen dos supuestos de exención: uno de carácter objetivo, para las actividades de transmisión de datos y provisión de acceso a una red de comunicaciones, ya que solo es necesaria la no modificación de los contenidos; y uno de carácter subjetivo, en el caso de almacenamiento y alojamiento de datos, ya que en estos casos el agente debe cumplir con una cierta diligencia en la prestación del servicio.

Queda manifiesto que la Ley solo se refiere al primero de los agentes, es decir, solo contempla a aquellos sujetos de la sociedad de la información que hayan intervenido como meros intermediarios en el tratamiento de los datos que almacenan y de los servicios que proporcionan. Por tanto, su función es únicamente poner en contacto a los consumidores y/o profesionales para que se produzca la oferta y la demanda, trabajando en un sistema en el que se permite a los interesados realizar las actividades de intercambio de bienes o servicios, ya sean C2C o B2C. El art. 13 de la LSSI hace referencia a los prestadores de servicios, pero solo para dejar claro que estos estarán sujetos a la responsabilidad civil, penal y administrativa establecida con carácter general en el ordenamiento jurídico, sin perjuicio de lo dispuesto en esta Ley. Así, en este caso, el régimen de responsabilidad o las exenciones de la misma no se aplicarán con la misma facilidad que para los meros intermediarios, en el caso de aquellas plataformas que actúen como ambos. Así, se hace necesaria la observación del caso concreto, analizando el hecho que ha desencadenado una posible responsabilidad por parte de la plataforma, y el papel que la misma haya tomado.

\subsection{LA GRAdUACión DE LA RESPONSABILIDAd EN BASE AL CONTROL DE LA PLATA- FORMA}

La Comunicación a la Comisión Una Agenda Europea ofrece una manera de distinguir entre los prestadores de servicios y los intermediarios digitales, y sin embargo la Ley no expone de manera precisa cuándo se trata de uno y cuándo de otro. Esta clara separación pone al descubierto a su vez a aquellas plataformas que comparten rasgos de ambas figuras. Así, en Una Agenda Europea, se fijan tres criterios para determinar el nivel de control de las plataformas:

a. Con respecto al precio: ¿la plataforma fija/no fija el precio final que deben pagar los usuarios por el servicio?

b. Con respecto a las condiciones contractuales: ¿establece términos y condiciones contractuales que determinarán la relación contractual entre el prestador de servicios y el usuario?

35 Peguera Ponch, M., Mensajes y mensajeros en internet: La responsabilidad civil de los proveedores de servicios intermediarios. 2001. 
c. Con respecto a la propiedad de activos clave: :posee activos para prestar el servicio subyacente?; ¿puede prestar el servicio sin necesidad de la actividad de los prestadores de servicio?

Cuando hacemos referencia a un proveedor de servicios nos referimos a un intermediario digital que ha comenzado a ofrecer el servicio subyacente presente en la plataforma. Como vemos, el criterio sobre el que se basa la diferenciación es la instrumentalidad de la propia plataforma.

En este sentido, la Sentencia de 10 de diciembre de 2019, Airbnb Ireland UC, C-390/18, EU:C:2019:1112, dictada por el Tribunal de Justicia de la Unión Europea (de ahora en adelante TJUE), versa, entre otros, sobre el carácter disociable o indisociable de la actividad principal (puesta en contacto de la oferta y la demanda) con respecto a la actividad secundaria (posible prestación del servicio subyacente, en este caso el alojamiento) y, por tanto, el papel de la plataforma hacia la intermediación o la prestación de servicios. Así, la sentencia falla sobre una cuestión prejudicial elevada por el Gobierno francés para la interpretación del artículo 3 de la DCE, relativo a determinados aspectos jurídicos de los servicios de la sociedad de la información en relación con el comercio electrónico en el mercado interior a raíz de una denuncia inicial por la cual se demanda a Airbnb Ireland por haber ejercido desde abril de 2012 hasta enero de 2017 una actividad de intermediación y gestión de inmuebles sin estar en posesión de la acreditación necesaria estipulada en la legislación francesa (tarjeta profesional de agente inmobiliario). El TJUE, para dilucidar sobre la interpretación del mencionado artículo 3 y su aplicabilidad al caso concreto, considera finalmente que Airbnb Ireland es un prestador de servicios de la sociedad de la información y que su actividad es la intermediación entre la oferta y la demanda; citando la propia sentencia: «ese servicio de intermediación es disociable de la transacción inmobiliaria propiamente dicha en la medida en que no solo tiene por objeto la realización inmediata de una prestación de alojamiento", sino también "proporcionar un instrumento que facilite la conclusión de contratos en futuras transacciones». Esta afirmación se basa sobre las siguientes premisas: la actividad de intermediación no es indispensable para llevar a cabo la actividad de prestación del servicio de alojamiento, es disociable de la transacción inmobiliaria objeto del contrato y no ejerce una influencia decisiva sobre las condiciones en las que se ha de desarrollar el servicio de alojamiento ${ }^{36}$, argumentos esgrimidos por la parte demandante para constatar que Airbnb Ireland presta «servicios adicionales característicos de la actividad de intermediación en las transacciones mobiliarias».

Como se observa, estos tres criterios a que aludíamos más arriba inciden de manera directa en el régimen de responsabilidad de las plataformas. La línea divisoria estará en si ejercen una actividad puramente auxiliar o si bien prestan el servicio que

${ }^{36} \mathrm{Si}$ bien es cierto que pone a disposición de los arrendadores de alojamientos servicio de fotografía, plantilla donde pueden definir el contenido del contrato de alojamiento, seguro de responsabilidad civil y garantía por daños, todos ellos de carácter opcional. 
pone en contacto a los usuarios (prestadores de servicio o no). Por lo tanto, cuanto mayor control ejerzan sobre los bienes o servicios que se transmitan a través de la propia plataforma, mayor análisis en cuanto a la graduación de la responsabilidad y posible conocimiento de la ilicitud de sus contenidos generarán. A este respecto, es muy interesante el planteamiento de Rodríguez de las Heras Ballell $(2010)^{37}$, pues supone un cambio en el método de aproximación al problema: en vez de determinar como ilícita una conducta ya realizada, plantea qué acciones pueden llevar a cabo las plataformas para paliar, atenuar o evitar los posibles riesgos derivados del contenido transmitido, alojado, enlazado, etc. Esta autora ofrece cinco estrategias:

1. «Informar al usuario del tipo de servicio que se presta y de las condiciones de prestación.

2. Implantar sistemas de detección y retirada y sistemas de reposición de contenidos retirados o bloqueados.

3. Recurrir preferentemente al uso de enlaces ordinarios.

4. Incluir en un lugar visible y de fácil acceso la política de supervisión y de retirada de los contenidos, aplicable muy especialmente a los casos de foros y chats moderados.

5. Incluir cláusulas razonables de limitación o exoneración de responsabilidad o, en todo caso, de advertencia a los usuarios de las limitaciones propias del servicio (warning terms)».

Todo lo señalado hasta ahora pone de relieve que son necesarios más criterios para poder diferenciar estos dos tipos de agentes intervinientes, ya que el régimen de responsabilidad puede ir desde una exoneración absoluta hasta una pena o multa de consideración, con el añadido de la complejidad que presenta para su tipificación las situaciones en las que un intermediario comienza a proveer del servicio subyacente.

\section{ALGUNAS CONCLUSIONES}

Las plataformas de economía colaborativa implican el nacimiento de un nuevo modelo de mercado nacido a raíz del desarrollo de las nuevas tecnologías de la información y la comunicación, la emergencia de la sociedad de la información y la crisis económica mundial de la última década. Nacida en una época que exigía solidaridad y empatía, la economía colaborativa se presentó como una necesidad de la sociedad para poder aliviar las cargas de las transacciones económicas cotidianas, lo que no excluye que se haya generado un negocio económico de grandes dimensiones que han aprovechado los profesionales del sector en cada caso.

37 Rodríguez de las Heras Ballell, T., «Responsabilidad jurídica de los marcos electrónicos». ICEX eMarketServices. 2010. Disponible en http://www.emarketservices.es/icex/cma/contentTypes/common/records/mostrarDocument o/?doc=4327620. 
Resulta prácticamente imposible para la legislación europea y nacional establecer un régimen de responsabilidad delimitado en el que se pueda subsumir toda la casuística. Es por ello por lo que la Directiva 2000/31/CE y su posterior transposición a la legislación española con la Ley 34/2002 solo se ocupa de las plataformas que actúan como intermediarias puras, ya que tienen unas actividades delimitadas que se han podido distinguir y regularizar. Ello ha dado como resultado que las plataformas que actúan como prestadoras de servicios queden al amparo de la legislación interna de cada país, ya que su casuística es mucho más variada y las actividades que realizan pueden ser de cualquier índole, siempre que exista oferta y demanda.

La LSSI se configuró como una ley que pretendía dar seguridad jurídica al nacimiento de las plataformas electrónicas y las posibles actividades que llevaban a cabo las mismas; sin embargo, a la fecha de publicación de dicha ley, debemos tener en cuenta que no existían plataformas tales como Youtube, Twitter, TikTok, Facebook, etc., plataformas que cambiaron el mercado digital existente en el momento, con respecto a tal y como lo conocemos ahora. Así, define nuevos conceptos, diferencia entre agentes de la sociedad de la información, establece exoneraciones de la responsabilidad, etc. Actualmente la seguridad jurídica que proporcionó ha desaparecido, ya que, al regular tan ambiguamente los preceptos de responsabilidad, no se entiende bien cuáles son los presupuestos para determinarla.

Actualmente la LSSI queda, en cierto sentido, incompleta y ambigua, el cambio del paradigma digital y su crecimiento exponencial plantean situaciones no contempladas en la ley, aunque no debemos dejar de lado que la DCE y su transposición a través de la LSSI han permitido el florecimiento y transformación de los servicios digitales de finales del pasado siglo. En este sentido se pronuncia la Comisión Europea con su comunicación sobre Online Platforms and the Digital Single Market Opportunities and Challenges for Europe ${ }^{38}$ sobre garantizar la conducta responsable de las plataformas en línea.

Aun así, la LSSI no contempla la responsabilidad de los proveedores de servicios, sino solo de los intermediarios que proporcionan la plataforma para el desarrollo de los mismos. El régimen de responsabilidad contractual y extracontractual español es aplicable siempre que no se den las excepciones que la LSSI considera para la exención de la responsabilidad.

Es necesario que el legislador se pronuncie a la hora de establecer criterios para determinar cuándo estamos ante un mero intermediario digital y cuándo estamos ante un real proveedor de servicios. Dejarlo al arbitrio de la legislación interna de cada país puede derivar en una desigualdad importante, pese a estar en el marco común de la Unión Europea. Se producen y se producirán situaciones de abuso de derecho, ya que en determinados territorios será más beneficioso ser un prestador

38 Comunicación de la Comisión al Parlamento Europeo, al Consejo, al Comité Económico y Social Europeo y al Comité de las Regiones: «Las plataformas en línea y el mercado único digital: Retos y oportunidades para Europa». Publicado en el Registro de documentos de la Comisión el 25 de mayo de 2016. Documento $\operatorname{COM}(2016) 288$ final. 
de servicios que en otros, o bien la justicia será más benevolente con la comisión de un ilícito digital en unos países que en otros, y así sucesivamente.

Con respecto a la determinación de la responsabilidad de los proveedores de servicios, entendiendo por tal el intermediario que proporciona el servicio subyacente, la pasividad se revela como un elemento clave para la determinación de la responsabilidad de las plataformas. Así, cuanto más intervenga la plataforma en la regulación de elementos esenciales del contrato, más sujeta estará al régimen de responsabilidad contractual y extracontractual estatal; de la misma manera, cuanto menos intervenga, más difícil será determinar la responsabilidad de la misma.

Recibido: enero de 2021; ACEPTADo: febrero de 2021 